\title{
Vibration Control of Flexible Link Manipulator Using SDRE Controller and Kalman Filtering
}

\author{
Mule PALA PRASAD REDDY ${ }^{1}$, Jeevamma JACOB ${ }^{2}$ \\ ${ }^{1}$ National Institute of Technology, Calicut, 673601, India \\ e-mail:prasadreddy.mule@gmail.com \\ ${ }^{2}$ National Institute of Technology, Calicut, 673601, India \\ e-mail:jeeva@nitc.ac.in
}

\begin{abstract}
The problem of estimating the flexural states while applying State-Dependent Riccati Equation (SDRE) technique to flexible link manipulator (FLM) is the focus of this paper. The proposed method investigates the effect of employing Kalman Filter as state estimator in the case of the deterministically modelled FLM. The dynamic model of the FLM is derived through combined Euler Lagrangian-Assumed modes approach based on two significant modes, resulting in a nonlinear model with six states. The information of states being crucial to the imple mentation of SDRE controller, the state estimator based on Kalman filter designed in this paper, minimizes the effect of noises that may corrupt the state measurements. Simulation results reveal the effectiveness of Kalman filter based SDRE controller for accurate positioning and vibration suppression of the FLM.
\end{abstract}

Keywords: Flexible Link Manipulator, Assumed modes methods, State dependent Riccati equation, Kalman Filter.

\section{Introduction}

The requirements of light weight and lower energy consumption in space applications motivate the utilization of flexible link robot manipulators. However, the flexible nature of the manipulator causes difficulty in obtaining an accurate model and makes the controller design very difficult.

Various modeling approaches have been proposed in the literature to derive the dynamic model of FLM, such as Lagrangian-Assumed modes method [16], finite element method [25], and Kane's approach [14]. A detailed approach to modeling of flexible manipulators using recursive Lagrangian dynamics is presented by Book [6] and Li and Sankar [8]. The kinematics of manipulator using homogeneous transformation matrices is presented in [15]. In [18], the dynamic model of FLM is approximated by considering only two flexible modes. The way of expressing the tip deflection as a function of mode shapes is presented in [21] and [23], which is necessary in Euler Bernoulli beam theory. However, the models derived from Lagrangian-assumed modes method are useful in controller design perspective. Hence, dynamic model of FLM based on combined Lagrangian-Assumed modes method is used in this work. The model is truncated with the first two significant modes resulting in a sixth order nonlinear model.

There are several control schemes applied in the past for trajectory tracking and vibration suppression of FLM based on nonlinear models. These control schemes include feedback linearization based techniques [7], Lyapunov function based control [12], recursive back stepping [9] and nonlinear $\boldsymbol{H}_{\infty}$ control [4]. SDRE based techniques provide a systematic method to design nonlinear controllers. The controller design for nonlinear systems via SDRE technique is presented in [2-3], which use a state-dependent coefficient (SDC) parameterization, to produce a constant state-space model. The general idea of the SDRE technique is presented in [10]. The issues in realization of SDRE scheme in real time are presented in [13]. Controllability test on SDC form carries significance in enabling the feasibility of the nonlinear optimal control. The connection between controllability of SDC parameterizations and exact system controllability is introduced in [17]. The efficiency of SDRE controller in terms of computational time is reported in [20]. For trajectory tracking of FLM, SDRE scheme is applied in real time with position variable as feedback in [1]. Simultaneous position as well as vibration control requires feedback of position and deflection variables. None of the existing SDRE based controllers has considered this aspect. Owing to the problems of noise and disturbance issues associated with the sensing of position and deflection variables, state estimator based on Kalman filters is an appropriate strategy to achieve tip position 
control with vibration suppression. In this paper Kalman filter based SDRE controller is proposed for the control of FLM.

Organisation of the article is as follows. Section 2 gives the modeling of flexible link manipulator by Lagrangian-AMM approach. Section 3 gives the design of SDRE controller and section 4 gives the Kalman filter design for flexural states estimation. Section 5 gives the formulation of SDC matrices. Section 6 gives the simulation results. Section 7 gives the conclusions.

\section{Modeling of Flexible-Link Manipulator}

The schematic diagram of FLM rotating in the horizontal plane and clamped at one end is shown in Figure 1. The flexible arm of length $l$, flexural rigidity $E I$ and mass density $\rho$ is joined to the hub of inertia $I_{H}$. Torque $\tau$ is applied at the hub.

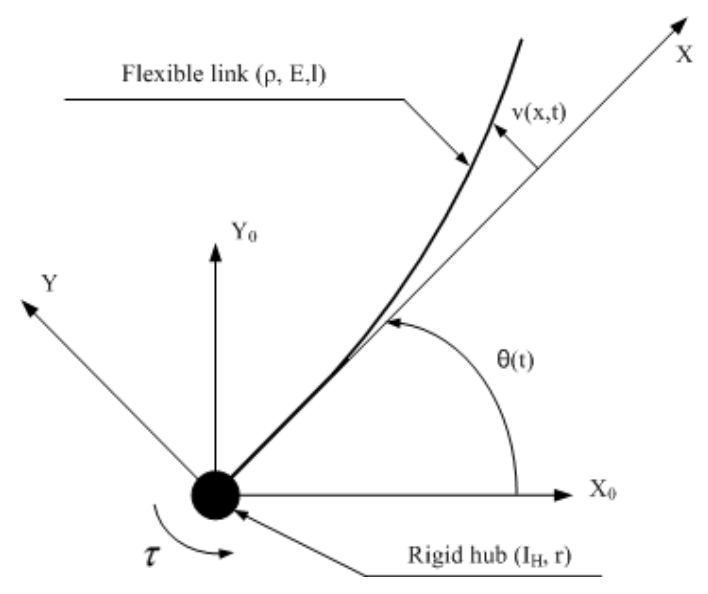

Figure 1. Representation of flexible-link manipulator

The net deflection $v(x, t)$ of the arm at a distance $x$ at time $t$, is expressed using combined Euler Lagrangian-AMM approaches and is given by

$$
v(x, t)=\sum_{i=1}^{m} \phi_{i}(x) \delta_{i}(t)
$$

where $m$ is a number of modes, $\phi_{i}(x)$ indicates mode function and $\delta_{i}(t)$ is model variable for an $i^{\text {th }}$ mode. In this work, the first two significant modes are considered while modeling.

\subsection{Lagrange's Equations of FLM}

The Kinetic energy owing to the motion of hub and link of the manipulator is given by

$$
T=\frac{1}{2} I_{H} \dot{\theta}^{2}+\frac{1}{2} \rho \int_{0}^{l} \dot{r}^{T} \dot{r} d x
$$

where $r$ is a position vector in (OXY) coordinates and $\theta(t)$ represents hub angle position. Then, $r(\theta, x, t)$ can be expressed as:

$$
r(\theta, x, t)=\left[\begin{array}{cc}
\cos \theta & -\sin \theta \\
\sin \theta & \cos \theta
\end{array}\right]\left[\begin{array}{c}
x \\
v(x, t)
\end{array}\right]
$$

The potential energy possessed in the arm due to the elastic deformation is expresses as

$$
V=\frac{1}{2} \int_{0}^{l} E I\left(\frac{\partial^{2} v}{\partial x^{2}}\right)^{2} d x
$$

The Lagrange's approach is used to obtain differential equations of motion of FLM. A system with $n$ coordinates has $n$ differential equations of the form:

$\frac{d}{d t}\left(\frac{\partial L}{\partial \dot{f}_{i}}\right)-\frac{\partial L}{\partial f_{i}}=F_{i} \quad i=1,2, \ldots . ., n$

where $F_{i}$ is generalized force action on generalized coordinates $f_{i}$ and the Lagrange's operator is defined by

$$
L=T-V
$$

Using equation (5) and (6), the differential equation of the form (7) can be obtained.

$$
\frac{d}{d t}\left(\frac{\partial T}{\partial \dot{f}_{i}}\right)-\frac{\partial T}{\partial f_{i}}+\frac{\partial V}{\partial f_{i}}=F_{i} \quad i=1,2, \ldots, n
$$

The generalized coordinate vector contains hub angle $(\theta(t))$ and modal variables $\left(\delta_{1}(t) \ldots\right.$ $\left.\delta_{\mathrm{m}}(t)\right)$ as its elements .Using the equations (2)(4) and (7), dynamics of FLM can be obtained in the form specified by

$\underbrace{M(\theta, \delta)}_{3 \times 3}\left[\begin{array}{c}\ddot{\theta} \\ \ddot{\delta}_{1} \\ \ddot{\delta}_{2}\end{array}\right]+\underbrace{\left[\begin{array}{l}h_{1}(\theta, \dot{\theta}, \delta, \dot{\delta}, t) \\ h_{2}(\theta, \dot{\theta}, \delta, \dot{\delta}, t) \\ h_{3}(\theta, \dot{\theta}, \delta, \dot{\delta}, t)\end{array}\right]}_{3 \times 1}+\underbrace{\left[\begin{array}{ccc}0 & 0 & 0 \\ 0 & k_{1} & 0 \\ 0 & 0 & k_{2}\end{array}\right]}_{3 \times 1} \underbrace{\left[\begin{array}{c}\theta \\ \delta_{1} \\ \delta_{2}\end{array}\right]}_{3 \times 3}=\underbrace{\left[\begin{array}{c}\tau \\ 0 \\ 0\end{array}\right]}_{3 \times 1}$

where $M \in R^{3 \times 3}$ represents the inertia matrix, $H=\left[\begin{array}{lll}h_{1} & h_{2} & h_{3}\end{array}\right]^{T}$ is a vector of centrifugal and Coriolis forces, $\tau$ is the control input torque, 
and $K \in R^{3 \times 3}$ is stiffness matrix. Since the deflections of the beam are small compared to link length, the output expresses in the normalized form:

$$
y=\theta(t)+\sum_{i=1}^{m} \phi_{i}(l) \delta_{i}(t)
$$

After substituting the physical parameters as given in Table 1 into (8), the manipulator dynamics can be obtained in the form:

$$
M \ddot{x}+C \dot{x}+K x=U
$$

where $M$ and $K$ are inertia and stiffness matrices respectively, $C \dot{X}$ represents Coriolis and centrifugal force terms, which are given by

$$
\begin{aligned}
M & =\left[\begin{array}{ccc}
m_{11} & -0.078 & -0.072 \\
-0.078 & 0.162 & 0.159 \\
-0.072 & 0.159 & 0.168
\end{array}\right] \\
m_{11} & =0.04+0.162 \delta_{1}^{2}+0.168 \delta_{2}^{2}+0.318 \delta_{1} \delta_{2} \\
C \dot{x} & =\left[\begin{array}{cc}
0.324 \delta_{1} \dot{\delta_{1}} \dot{\theta}+0.336 \delta_{2} \dot{\delta}_{2} \dot{\theta}+0.318 \delta_{2} \dot{\delta}_{1} \dot{\theta} \\
-0.162 \delta_{1} \dot{\theta}^{2}-0.159 \delta_{2} \dot{\theta}^{2}+747.72 \delta_{2} \\
-0.159 \delta_{1} \dot{\theta}^{2}-0.168 \delta_{2} \dot{\theta}^{2}+747.72 \delta_{1}
\end{array}\right] \\
K & =\left[\begin{array}{ccc}
0 & 0 & 0 \\
0 & 247.72 & 0 \\
0 & 0 & 3040.92
\end{array}\right] \\
U & =\left[\begin{array}{ccc}
\tau & 0 & 0
\end{array}\right]^{T}
\end{aligned}
$$

The normalized output is given by $y(t)=\theta-2.63 \delta_{1}-3.69 \delta_{2}$

Table 1. Physical Parameters of FLM [22]

\begin{tabular}{|l|l|}
\hline \multicolumn{1}{|c|}{ Parameter } & \multicolumn{1}{c|}{ Value } \\
\hline Beam length $(l)$ & $0.9 \mathrm{~m}$ \\
\hline Beam width $(w)$ & $0.0032 \mathrm{~m}$ \\
\hline Beam height $(h)$ & $0.019 \mathrm{~m}$ \\
\hline Young's modulus $(E)$ & $71 \times 10^{9} \mathrm{~Pa}$ \\
\hline Moment of inertia $(I)$ & $5.253 \times 10^{-11} \mathrm{~m}^{4}$ \\
\hline Hub inertia $\left(I_{H}\right)$ & $5.8598 \times 10^{-4} \mathrm{kgm}^{2}$ \\
\hline Mass density $(\rho)$ & $2710 \mathrm{~kg} / \mathrm{m}^{3}$ \\
\hline
\end{tabular}

\subsection{State-Space Formulation}

It is convenient to express dynamic model in state-space form for controller design. By choosing $\left[\theta, \dot{\theta}, \delta_{1}, \dot{\delta}_{1}, \delta_{2}, \dot{\delta}_{2}\right]$ as state variables, the state equations of the model (10) can be written as follows:

$$
\begin{aligned}
\dot{x}_{1}= & x_{2} \\
\dot{x}_{2}= & \frac{1}{D}\left[110.32 x_{3} x_{2}^{2}+101.842 x_{5} x_{2}^{2}-\right. \\
& 458.289 x_{2} x_{3} x_{4}-475.263 x_{5} x_{6} x_{2} \\
& -449.8 x_{4} x_{5} x_{2}-449.8 x_{3} x_{6} x_{2} \\
& +746784.736 x_{5}+103514.47 x_{3} \\
& +1414.474 u] \\
\dot{x}_{3}= & x_{4} \\
\dot{x}_{4}= & \frac{1}{D}\left[95.421 x_{3} x_{2}^{2}+87.157 x_{5} x_{2}^{2}-\right. \\
& 392.21 x_{2} x_{3} x_{4}-406.73 x_{5} x_{6} x_{2}- \\
& 384.94 x_{4} x_{5} x_{2}-384.94 x_{3} x_{6} x_{2} \\
& +825318.59 x_{5}+128523.85 x_{3}+ \\
& 237.63 x_{3} x_{5}^{2} x_{2}^{2}+449.8 x_{5} x_{3}^{2} x_{2}^{2}+ \\
& 229.144 x_{3}^{2} x_{2}^{2}+60631741.32 x_{3}^{2} x_{5} \\
& +93247410 x_{3} x_{5}^{2}+9150832895 x_{3}^{3}+ \\
& \left.4424932842 x_{5}^{3}+1210.526 u\right] \\
\dot{x}_{5}= & x_{6} \\
\dot{x}_{6}= & \frac{1}{D}\left[-42.078 x_{3} x_{2}^{2}-37.842 x_{5} x_{2}^{2}+\right. \\
& 174.89 x_{2} x_{3} x_{4}+181.263 x_{5} x_{6} x_{2}+ \\
& 171.552 x_{4} x_{5} x_{2}+171.552 x_{3} x_{6} x_{2} \\
& -479214.73 x_{5}-81726.140 x_{3}+ \\
& 449.8 x_{3} x_{5}^{2} x_{2}^{2}+229.144 x_{5} x_{3}^{2} x_{2}^{2}+ \\
& 237.63 x_{5}^{2} x_{2}^{2}-63546931.84 x_{3}^{2} x_{5} \\
& -97478194.47 x_{3} x_{5}^{2}-9680494.73 x_{3}^{3} \\
& \left.-46194274.74 x_{5}^{3}-539.473 u\right] \\
& \\
&
\end{aligned}
$$

where

$$
D=\left(76+17414 x_{3}^{2}+18060 x_{5}^{2}+34185 x_{3} x_{5}\right)
$$

The state equations written in general statespace form are as follows:

$\dot{x}=f(x)+g(x) u$

where $f(x)$ and $g(x)$ are vectors of nonlinear elements and $u$ is the control input applied to the manipulator $(u=\tau)$.

\section{SDRE Regulator Problem}

Consider the general form of infinite-horizon nonlinear regulator problem that minimizes the performance measure [11]: 


$$
J=\int_{0}^{\infty}\left(x^{T} Q(x) x+u^{T} R(x) u\right) d t
$$

with respect to the system state $x$ and control input $u$ subject to the nonlinear differential constraint:

$$
\dot{x}=f(x)+g(x) u
$$

The objective is to find an approximate solution of problem (12)-(13) of the type $u(x)=-k(x) x$, where $k$ is a nonlinear function of $x$. SDRE nonlinear control approach is similar to the LQR approach for linear systems [24], as a result the system equations are transformed to SDC form:

$\dot{x}=A(x) x+B(x) u$

where $f(x)=A(x) x$ and $g(x)=B(x)$. The selection of $A(x)$ is not exceptional and the parameterization is achievable only if $f(0)=0$ and $f(x)$ is continuously differentiable. The parameterization of nonlinear system is performed by checking pointwise observability and controllability [5]. Once the SDC matrices are formed, then the state feedback control law is obtained in the form

$$
u(x)=-k(x) x=-R^{-1}(x) B^{T}(x) P(x) x
$$

where $P(x)$ is the solution of state-dependent Riccati equation:

$$
\left.\begin{array}{l}
P(x) A(x)+A^{T}(x) P(x)- \\
P(x) B(x) R^{-1}(x) B^{T}(x) P(x)+Q(x)=0
\end{array}\right\}
$$

The stabilization and performance of the system depend on the choice of weighting matrices $Q$ and $R$. These matrices are taken as diagonal. To impose some restriction on a state, the corresponding entry in $Q$ should be weighted more. Also, as the value of $R$ increases the feedback gain decreases, resulting in a sluggishness response.

\section{Kalman Filter Technique for State Estimation}

In this section Kalman filtering techniques for estimation of flexural states is presented. The Kalman filter based SDRE is formulated by constructing the twofold of SDRE control design and resulting in a steady-state linear
Kalman filter structure. This brings the given nonlinear system into linear structure in SDC form.

Let us consider the stochastic nonlinear system

$$
\left.\begin{array}{l}
\dot{x}=f(x)+g(x) u+K w \\
y=h(x)+v
\end{array}\right\}
$$

where $K$ is a weight matrix of process noise with appropriate dimensions, $w$ is white process noise and $v$ is white measurement noise. Now equation (17) is written in the SDC form:

$$
\left.\begin{array}{l}
\dot{x}=F(x) x+G(x) u+K w \\
y=H(x) x+v
\end{array}\right\}
$$

where $F(x) x=f(x), G(x)=g(x)$ and $H(x) x=h(x)$.

A state observer exists for a class of systems, which are observable. A test for observability of nonlinear systems of the form shown in equation (17) is given by Isidori in [19], it shows that the following is true in an observable nonlinear system:

$\operatorname{rank}\left[\begin{array}{c}d h(x) \\ d L_{f} h(x) \\ \vdots \\ d L_{f}^{n-1} h(x)\end{array}\right]=n$

where $L_{f}(x)$ is the Lie derivative of $h(x)$. Here observability of the system is assumed in nonlinear sense and the pointwise observability can be checked in the region of interest. The Kalman filter equations for states estimation in the SDRE design [11] is as follows

$\dot{\hat{x}}=F(\hat{x}) \hat{x}+G(\hat{x}) u+k_{f}(\hat{x})[y(x)-H(\hat{x}) \hat{x}]$

where $k_{f}(\hat{x})=\Gamma H^{T}(\hat{x}) V^{-1}$ and $\Gamma \quad$ is the positive definite solution of the following equation

$$
F(\hat{x}) \Gamma+\Gamma F^{T}(\hat{x})-\Gamma H^{T}(\hat{x}) V^{-1} H(\hat{x}) \Gamma+K W K^{T}=0
$$

The matrices $W$ and $V$ are chosen as the covariance matrices [11] for the corrupting noise terms such that

$$
\left.\begin{array}{l}
E\left[w(t) w^{T}(t+\tau)\right]=W(t) \delta(\tau) \\
E\left[v(t) v^{T}(t+\tau)\right]=V(t) \delta(\tau)
\end{array}\right\}
$$




\section{SDC Formulation}

The SDRE method depends upon the ability of writing the constraint dynamics (11) in a pointwise linear structure, having SDC form:

$$
\dot{x}=\underbrace{\left[\begin{array}{cccccc}
0 & 1 & 0 & 0 & 0 & 0 \\
0 & A_{22} & A_{23} & A_{24} & A_{25} & A_{26} \\
0 & 0 & 0 & 1 & 0 & 0 \\
0 & A_{42} & A_{43} & A_{44} & A_{45} & A_{46} \\
0 & 0 & 0 & 0 & 0 & 1 \\
0 & A_{62} & A_{63} & A_{64} & A_{65} & A_{66}
\end{array}\right]}_{A(x)}\left[\begin{array}{c}
x_{1} \\
x_{2} \\
x_{3} \\
x_{4} \\
x_{5} \\
x_{6}
\end{array}\right]+\underbrace{\left[\begin{array}{c}
0 \\
H_{11} / k \\
0 \\
H_{21} / k \\
0 \\
H_{31} / k
\end{array}\right]}_{B(x)} u
$$

$Y=\left[\begin{array}{llllllllllll}1 & 0 & -2.63 & 0 & -3.69 & 0\end{array}\right]\left[\begin{array}{llllll}x_{1} & x_{2} & x_{3} & x_{4} & x_{5} & x_{6}\end{array}\right]^{T}$ The elements of $A(x)$ and $B(x)$ are as follows:

$A_{22}=\frac{1}{Z}\left(H_{12} f_{1}+H_{13} f_{2}\right), A_{23}=\frac{1}{Z}\left(H_{12} f_{3}+H_{13} f_{4}\right)$,

$A_{24}=\frac{1}{Z}\left(H_{11} f_{5}\right), A_{25}=\frac{1}{Z}\left(H_{12} f_{6}+H_{13} f_{7}\right)$,

$A_{26}=\frac{1}{Z}\left(H_{11} f_{8}\right), A_{42}=\frac{1}{Z}\left(H_{22} f_{1}+H_{23} f_{2}\right)$,

$A_{43}=\frac{1}{Z}\left(H_{22} f_{3}+H_{23} f_{4}\right), A_{44}=\frac{1}{Z}\left(H_{21} f_{5}\right)$,

$A_{45}=\frac{1}{Z}\left(H_{22} f_{6}+H_{23} f_{7}\right), A_{46}=\frac{1}{Z}\left(H_{21} f_{8}\right)$.

$A_{82}=\frac{1}{Z}\left(H_{32} f_{1}+H_{33} f_{2}\right), A_{63}=\frac{1}{Z}\left(H_{32} f_{3}+H_{33} f_{4}\right)$

$A_{84}=\frac{1}{Z}\left(H_{31} f_{5}\right), A_{85}=\frac{1}{Z}\left(H_{32} f_{6}+H_{33} f_{7}\right), A_{66}=\frac{1}{Z}\left(H_{31} f_{8}\right)$

$Z=76+17414 x_{3}^{2}+18060 x_{5}^{2}+34185 x_{3} x_{5}$

$H_{11}=107500, H_{12}=92000=H_{21}, H_{13}=-41000=H_{31}$

$H_{22}=266666\left(32+567 x_{3}^{2}+588 x_{5}^{2}+1113 x_{3} x_{5}\right)$

$H_{23}=-333.33\left(124+4293 x_{3}^{2}+4452 x_{5}^{2}+8427 x_{3} x_{5}\right)$

$H_{32}=H_{23}, H_{33}=2000\left(11+729 x_{3}^{2}+756 x_{5}^{2}+1431 x_{3} x_{5}\right)$

$f_{1}=0.162 x_{2} x_{3}+0.159 x_{2} x_{5}, f_{3}=-247.72$,

$f_{2}=0.159 x_{2} x_{3}+0.168 x_{2} x_{5}, f_{4}=-747.74$

$f_{5}=-0.324 x_{2} x_{3}-0.318 x_{2} x_{5}, f_{6}=-747.74$

$f_{7}=-3050.92, f_{8}=-0.336 x_{2} x_{5}-0.318 x_{2} x_{3}$

\section{Simulation Results}

In this section, simulations are carried out to demonstrate the control performance of Kalman filter based SDRE controller for flexible link manipulator. The system parameters are specified in Table land simulations were performed in MATLAB environment.

\subsection{SDRE Controller}

SDRE controller is used to achieve good tracking performance and suppression of vibrations at end point of FLM. A change in the step signal from $\theta=0^{\circ}$ to $\theta=90^{\circ}(1.57 \mathrm{rad})$ is taken as reference. The weighting matrices are taken as shown below.

$$
\begin{aligned}
& R(x)=2 \\
& Q(x)=\operatorname{diag}\left[\beta_{1}, \beta_{2}, \beta_{3}, \beta_{4}, \beta_{5}, \beta_{6}\right] \\
& \text { where } \quad \beta_{1}=\left(1+10 x_{1}^{2}\right), \beta_{2}=\left(1+10 x_{2}^{2}\right), \\
& \beta_{5}=\left(1+100 x_{3}^{2}\right), \beta_{4}=\left(1+10 x_{4}^{2}\right) \\
&
\end{aligned}
$$

Figure 2 show the control input, hub angle response shown in Figure 3 and first and second mode of vibrations are shown in Figure 4 and Figure 5 respectively. The hub angle response shows that the manipulator reaches the steady state value in 4 seconds without overshoot. Oscillations are damped out in 1.25 sec in first and second mode.

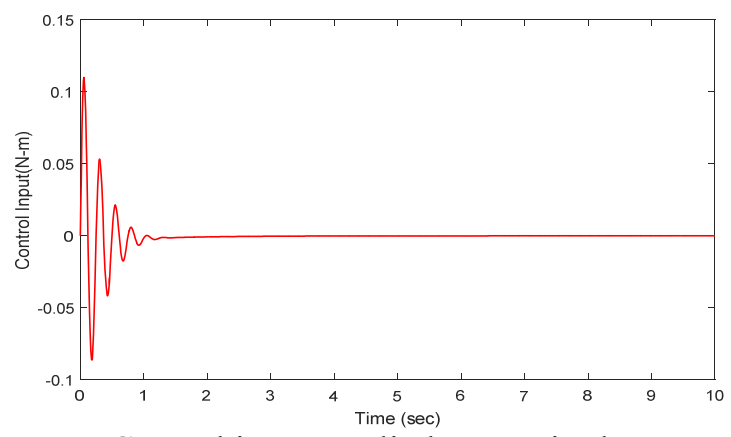

Figure 2.Control input applied to manipulator

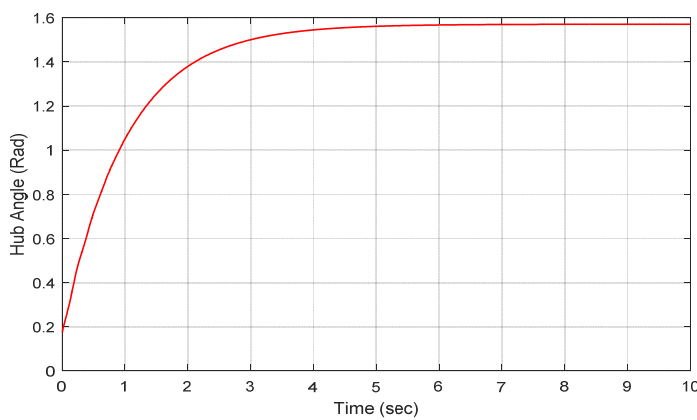

Figure 3.Hub angle response using SDRE controller 


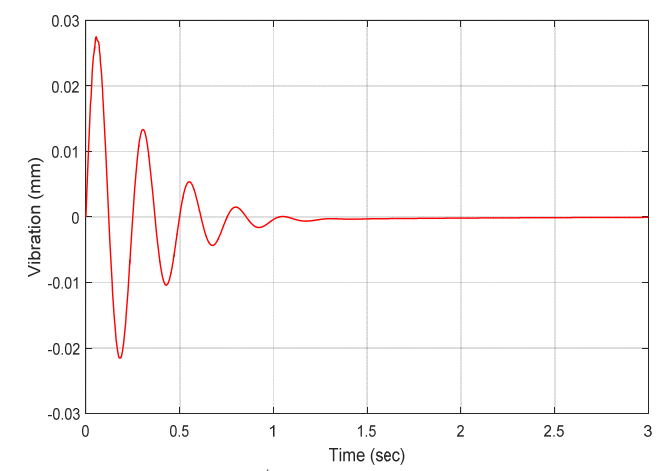

Figure 4. $1^{\text {st }}$ mode of vibration using SDRE controller

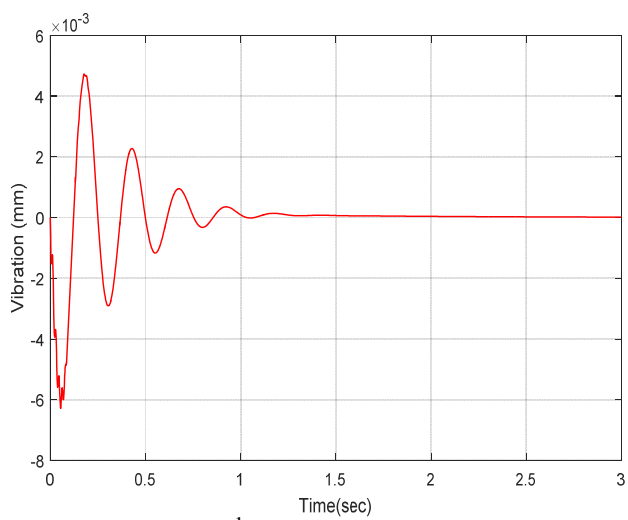

Figure 5. $2^{\text {nd }}$ mode of vibration using SDRE controller

\subsection{Kalman Filter based SDRE controller}

The assessment of the ability of Kalman filter to give good estimation of states in the presence of process and measurement noise is discussed in this section. The intensity of process noise is taken as $W=0.5$ and the intensity of measurement noise $V$ is taken as

$$
V=\left[\begin{array}{ccc}
0.1 & 0 & 0 \\
0 & 0.3 & 0 \\
0 & 0 & 1.5
\end{array}\right]
$$

Responses are obtained for two cases: (i). with estimation of hub angle (position) only and (ii). with estimation of hub angle as well as deflection variables. Figure 6-Figure 8 show the hub angle response, $1^{\text {st }}$ and $2^{\text {nd }}$ mode of vibration responses for this two cases.

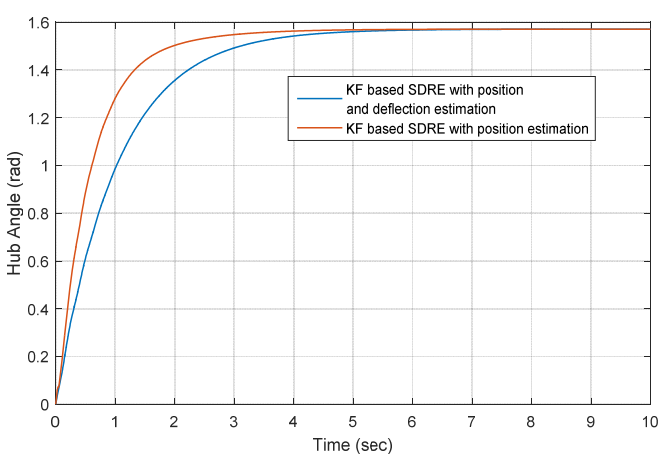

Figure 6.Hub angle response (Kalman filter based SDRE)

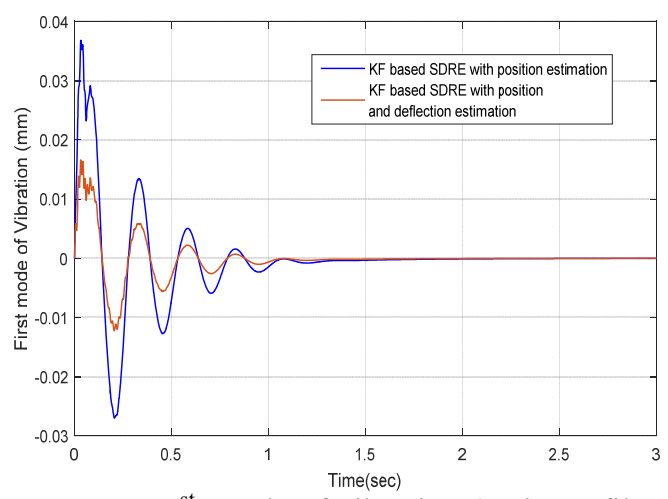

Figure 7. $1^{\text {st }}$ mode of vibration (Kalman filter based SDRE)

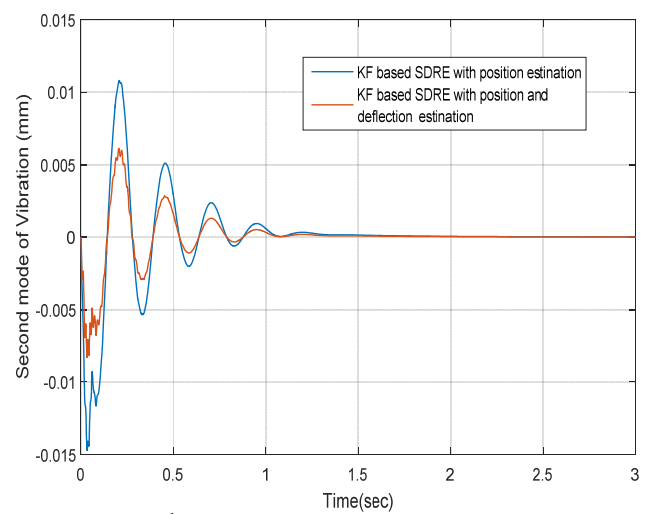

Figure 8. $2^{\text {nd }}$ mode of vibration (Kalman filter based SDRE)

These figures illustrate that even though faster hub angle response is obtained for case (i), the amplitude of vibrations are reduced considerably, for case (ii). With the improvements achieved by the Kalman filter based SDRE controller using the estimation of hub angle as well as deflection variables, it is assessed that the proposed controller qualifies to be adequate for simultaneous tracking and vibration suppression of FLM. 


\section{Conclusions}

The dynamic model of FLM has been derived by considering the first two significant modes using AMM approach. Good tracking performance as well as damping of deflections was achieved by the Kalman filter based SDRE controller. Furthermore, the advantage of estimating tip position as well as the first and second mode of vibrations instead of tip position alone was demonstrated through simulations. Due to the complexity of model dynamics only the manipulator with one link is considered in this work, and this technique may be extended further to multi link manipulators also.

\section{REFERENCES}

1. A. Shawky, D. Zydek, Y. Z Elhalwagy \& A. Ordys, "Modeling and nonlinear control of flexible link manipulator", Applied Mathematical Modeling (Elsevier), 37 (2013).

2. A. Shawky, A. Ordys, \& M. J. Grimble "Modeling and Control Design of a Flexible Manipulator Using SDRE Techniques" Proceeding of the 7th IEEE International Conference, MMAR 2001, August 2001.

3. A. Shawky, A. Ordys \& M. J. Grimble, "End-Point control of a flexible-link manipulator using $\mathrm{H} \infty$ nonlinear control via a state-dependent Riccati equation.", IEEE International Conference on Control Applications, September 2002.

4. Ball, J. A., J. W. Helton \& M. L. Walker, "Hळ Control for nonlinear Systems via Output Feedback," IEEE Transaction on Automatic Control, AC-38, 546559 (1993).

5. Banks, H. T, B. M. Lewis \& H. T. Tran, "Nonlinear feedback controllers and compensators: a state-dependent Riccati equation approach", Computational Optimization and Applications (springer link), June 2007, volume 37, Issue 2, pp 177-218.

6. Book, W. J, "Recursive Lagrangian dynamics of flexible manipulator arms," The Int. J. of Robotics Research, vol. 3, no. 3, pp. 87-101, Fall 1984.
7. Cannon, R. H. \& E. Schmitz, "Initial experiments on end-point control of a flexible one-link robot," The International Journal of Robotics Research 3 (3) (1984) $62-75$.

8. Chang-Jin Li \& T. Sankar, "Systematic Methods for Efficient Modeling and Dynamics Computation of Flexible Robot Manipulators," IEEE trans. on Syst., Man, Cybern., vol. 23, no. 1, pp. 77-95, Jan 1993.

9. Chiman Kwan \& F. L. Lewis, "Robust Backstepping Control of Nonlinear Systems using Neural Networks", IEEE Transactions on Systems, Man and Cybernatics, vol 30, No 6, November 2000.

10. Cloutier, J. R, "State Dependent Riccati Equation Techniques: An Overview", Proc. of American Control Conference, Mexico, 1997.

11. Curtis P. Mracek, Cloutier, James, R., D'Souza \& Christopher A., "A New Technique for Nonlinear Estimation", Proceedings of the 1996 IEEE International Conference on Control Applications Dearborn, September 15-18, 1996.

12. Dogan. K. M, E. Tatlicioglu, E. Zergeroglu \& K. Cetin, "Lyapunov-based output feedback learning control of robot manipulators", 2015 American Control Conference (ACC), Chicago, IL, 2015, pp. 5337-5342.

13. Erdem, E. B, "Analysis and Real time Implementation of State Dependent Riccati Equation Controlled Systems", $\mathrm{PhD}$ Thesis, University of Illinois at Urbana-Champaign, 2001.

14. Everett, L. J, "An extension of Kane's method for deriving equations of flexible manipulators", In Proceedings of the IEEE Conference on Robotics and Automation, pp. 391-398, 1989.

15. Fu, K. S, R. C. Gonzalez, C.S.G. Lee, Robotics. Singapore: McGraw Hill, 1987.

16. Geniele. H, Patel. R. V, \& Khorasani. K, "End-point control of a single link flexible manipulator: theory and experiments", 
IEEE Transactions on Control Systems Technology, 5, 556-560, 1997.

17. Hamett, K. D, Hall, C. D \& Ridgely, D.B. "Controllability issues in nonlinear state dependent Riccati Equation Control", Air Force Institute of Technology, WrightPatterson Air Force Base, Ohio.

18. Hasting, G. G, \& Book W. J, "A linear dynamic model for flexible robot manipulators", IEEE Control Syst. Mag., Vol. 7, pp. 61-64, 1987.

19. Isidori, A, Nonlinear Control Systems (Third Edition). Berlin: SpringerVerlag, 1995.

20. Katsev, S, "Streamlining of the State Dependent Riccati Equation Controller Algorithm for an Embedded Implementation", M.S Thesis, Rochester Institute of Technology, USA, 2006.

21. Martins, J. M., Z. Mohamed \& M. O. Tokhi "Approaches for dynamic modeling of flexible manipulator systems," IEE Proc.-Control Theory Appl., Vol. 150, No. 4, July 2003.
22. Pala Prasad Reddy, M., Shihabudheen K.V. \& Jeevamma Jacob, "Precise Nonlinear Modeling of Flexible Link Flexible Joint Manipulator," International Review on Modeling and Simulation, ISSN 1974-9821,vol. 5, no. 3, pp. 13681274, June 2012.

23. Rush, D. R, Dohramann C, G. Richard, et al, "Flexible Robot Dynamics and Controls," IFSR International Series on Systems Science and Engineering, vol.19, New York: KA/PA, 2002.

24. Shihabudheen, K. V., Jose Thankachan \& Chinmay Vasista,"SDRE control of flexible beam manipulator", 2015 International Conference on Soft Computing Techniques and Implementations (ICSCTI), 2015.

25. Usoro, P. B, Nandira. R, \& Mahil, S. S, "A finite element/Lagrange approach to modeling lightweight manipulators", Transactions of the ASME Journal of Dynamics Systems, Measurement and Control, 108, 198-205, 1986. 